\title{
Fluorescence Resonance Energy Transfer-based Biosensor Composed of Nitrogen-doped Carbon Dots and Gold Nanoparticles for the Highly Sensitive Detection of Organophosphorus Pesticides
}

\author{
Nian Chun Gong, Yan Le Li, Xi Jiang, Xiao Fang Zheng, Ya Ya WAng, and Shuang Yan Huan ${ }^{\dagger}$ \\ State Key Laboratory for Chemo/Biosensing and Chemometrics, College of Chemistry and Chemistry Engineer, \\ Hunan University, Changsha, 410082, China
}

\begin{abstract}
The present article reports a novel biosensor for organophosphorus pesticides based on fluorescence resonance energy transfer (FRET) between nitrogen-doped carbon dots (NC-dots) and gold nanoparticles (AuNPs). The effective NC-dots/ AuNPs assembly through the Au-N interaction results in good fluorescence quenching. Active acetylcholinesterase (AChE) catalyzes the hydrolysis of acetylthiocholine into -SH containing thiocholine to replace the NC-dots and trigger the aggregation of AuNPs. In the presence of paraoxon, the activity of AChE is inhibited, and thus preventing the generation of thiocholine, causing fewer NC-dots to be replaced. As a consequence, the fluorescence intensity gradually decreases with increasing amount of paraoxon. This biosensor does not require any complex synthesis or modification, and the results show a wide detection range of from $10^{-4}$ to $10^{-9} \mathrm{~g} / \mathrm{L}$ with a detection limit of $1.0 \times 10^{-9} \mathrm{~g} / \mathrm{L}(3.6 \times$ $10^{-12} \mathrm{~mol} / \mathrm{L}$ ). Two linear response regions have been reported with a turning point at about $10^{-6} \mathrm{~g} / \mathrm{L}$ and three different factors that would influence the response behavior. These phenomena discussed in detail so as to explain the special response mechanism.
\end{abstract}

Keywords Biosensor, nitrogen-doped carbon dots, fluorescence resonance energy transfer, organophosphorus pesticides

(Received March 17, 2016; Accepted May 23, 2016; Published September 10, 2016)

\section{Introduction}

Organophosphates (OPs) are widely used as pesticides in agriculture because of their relatively low persistence and high insecticidal activity. ${ }^{1,2}$ However, the widespread use of pesticides has resulted in serious environmental pollution, even lead to food safety issues..$^{2-4}$ Organophosphorus pesticides exhibit high toxicity on human health even at very low levels; for example, the median lethal dose of parathion is less than $10 \mathrm{mg} / \mathrm{kg}$. Hence, it is important to develop effective, low-cost and simple methods for detecting organophosphorus pesticides. ${ }^{2}$

In recent years, considerable efforts have been devoted to develop electrochemical, ${ }^{5-9}$ colorimetric ${ }^{10-12}$ or fluorescence biosensors $^{13-25}$ based on AchE-enzyme-inhibition strategy for OPs detection, in which the OPs could inhibit the activity of acetylcholinesterase (AChE), and thus prevent hydrolysis reaction of acetylcholine to choline. ${ }^{26-28}$ Among numerous analytical strategies, a fluorescence assay exhibits some advantages, such as high sensitivity and stability, as well as a fast response. Novel nanomaterials with unique chemical and physical properties have been widely employed to develop fluorescence assay for Ops, such as quantum dots (QDs), ${ }^{16,18,21,24,25}$ nanocrystal, ${ }^{17,19,20}$ graphene, ${ }^{22}$ and metal organic frameworks. ${ }^{23}$ Based on the AChE-inhibition strategy, Liu et al. ${ }^{13}$ presented a sensitive rhodamine B-covered gold nanoparticle (AuNPs)-

† To whom correspondence should be addressed.

E-mail: shuangyanhuan@163.com based assay with dual readouts for detecting OPs and carbamate pesticides. The fluorescent readout was a "signal-off" type assay based on released organic dyes by thiocholine through replacement; therefore, the detection limit of the fluorescent and naked eyes were identical $(0.1 \mu \mathrm{g} / \mathrm{L})$. Guo et al. ${ }^{14}$ employed CdTe QDs instead of organic dyes to construct a fluorescent method for the detection of OPs using an inner filter effect (IFE) of AuNPs on CdTe QDs. However, IFE would occur effectively only if the emission spectrum of the donor and the absorption spectrum of the acceptor were completely overlapped, which could restrict the choice of a suitable fluorophore..$^{29}$ The poor overlap between AuNPs and CdTe QDs influenced the IFE, and they reported a detection limit of only $2 \mu \mathrm{g} / \mathrm{kg}$. Based on the fluorescence resonance energy transfer (FRET) between upconversion nanoparticles and AuNPs, Long et al. ${ }^{15}$ demonstrated another highly sensitive assay that could detect OPs at the ng/L level. The only defect was the slightly complicated synthesis process of the quantum dots and upconversion nanoparticles. However, in the last two studies, the nanoparticles were adsorbed on the surface of AuNPs via electrostatic interactions, resulting in the quenching of fluorescence. As we know, acetylthiocholine also contains positive charges that may interfere the electrostatic interaction between nanoparticles and AuNPs, and possiblely influence the fluorescent stability.

Recently, carbon dots (C-dots) as a new class of fluorescent nanoparticles, have attracted wide attention in many different fields. ${ }^{30-36}$ Doping C-dots with nitrogen could effectively improve the fluorescence quantum yields (QY). ${ }^{31,32,35}$ 
The nitrogen-doped C-dots (NC-dots) are surrounded by $\mathrm{N}-\mathrm{H}$ groups, which is convenient for continuous chemical modification that widens its range of application. ${ }^{33,35}$ Deng et al. ${ }^{36}$ developed a novel nanosensor that was constructed by NC-dots and AuNPs to discriminate cysteine with multiple signals. Their results showed that the interactions between NC-dots and AuNPs were more efficient than that of the electrostatic interaction, and provided new strategies to design fluorescent biosensors for detecting OPs based on FRET.

In this work, we designed a novel FRET biosensor based on NC-dots/AuNPs nanoassembly for the highly sensitive detection of paraoxon, a commonly used type of OPs pesticide. The NC-dots with excellent fluorescence were prepared by a simple one-step microwave-assisted synthesis process. The effective assembly of the NC-dots "shell" on the surface of AuNPs through N-H groups resulted in good fluorescence quenching. AChE could catalyze the hydrolysis of acetylthiocholine to produce thiocholine, which is positively charged and bears an additional thiol group (-SH). Thiocholine can substitute the NC-dots and bind onto the surfaces of AuNPs, while inducing the aggregation of AuNPs and the fluorescence recovery of NC-dots simultaneously. In the presence of OPs, the activity of $\mathrm{AChE}$ is inhibited by pesticides, while preventing the generation of thiocholine, and thus fewer of NC-dots are replaced. As a consequence, the fluorescence intensity decreases gradually with the increasing amount of OPs. This biosensor does not require any complex synthesis or modification, and the results show a wide detection range from $10^{-4}$ to $10^{-9} \mathrm{~g} / \mathrm{L}$ with a detection limit of $1.0 \times 10^{-9} \mathrm{~g} / \mathrm{L}$. Two linear response regions have been reported with a turning point at about $10^{-6} \mathrm{~g} / \mathrm{L}$; three different factors that could influence the response behavior are discussed in detail to explain the special response mechanism.

\section{Experimental}

\section{Reagents and chemicals}

All chemicals were obtained from commercial suppliers, were of analytical grade, and used without any further purification. Acetylcholinesterase (AChE, from Electrophorus electricus), and acetylthiocholine iodide (ATI) were provided by SigmaAldrich (Shanghai, China). Paraoxon was obtained from agroenvironmental protection institution, of the ministry of agriculture (Tianjing China). Millipore Milli-Q ultrapure water (resistance $>18 \mathrm{M} \Omega \cdot \mathrm{cm}^{-1}$ ) was used throughout the experiments.

\section{Apparatus}

The UV absorption spectra were recorded with a UV-2450 spectrophotometer (Shimadzu, Japan). Fluorescence spectra were recorded on an F-7000 fluorescence spectrophotometer (Hitachi, Japan). The Fourier transform infrared (FT-IR) spectra were measured on a Nicolet 6700 FT-IR spectrometer (Thermo Scientific, US). Transmission electron microscopy (TEM) images were collect from a JEOL-1230 transmission electronic microscope (JEM-2010, Jeol, Japan). A dialysis bag (America Viskase) with a 500Da molecular weight cut-off was used to purify the NC-dots.

\section{Preparation of NC-dots/AuNPs assemblies}

The preparation of NC-dots/AuNPs assemblies is very simple. The NC-dots were prepared according to a report. ${ }^{32}$ AuNPs were prepared by using citrate sodium as a reducing reagent. ${ }^{37}$ Briefly, a stock solution of purified NC-dots $(50 \mu \mathrm{L}, 2.745 \mathrm{mg} / \mathrm{L})$ was added into solutions of the as-prepared AuNPs $(50 \mu \mathrm{L}$, $45 \mathrm{nmol} / \mathrm{L}$ ), which were prepared in a $10 \mathrm{mM}$ HEPES buffer

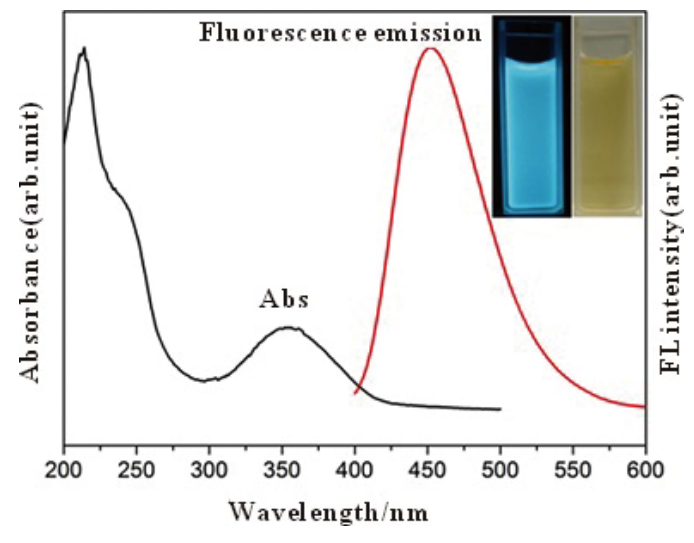

Fig. 1 UV-vis absorption spectrum and fluorescence emission spectrum of NC-dots. The inset pictures show the photographs of NCdots solution under UV (365 nm) and visible light.

( $\mathrm{pH}$ 7.4). The assembly process of the NC-dots on AuNPs could be completed within $10 \mathrm{~min}$, and then the fluorescence spectra were measured.

\section{Detection of pesticide}

AChE $(30 \mu \mathrm{L}, 0.1 \mathrm{U} / \mathrm{mL})$ was first mixed with various amounts of paraoxon (the final concentrations were $10^{-4}$ to $\left.10^{-9} \mathrm{~g} / \mathrm{L}\right)$, which were kept at $37^{\circ} \mathrm{C}$ for $20 \mathrm{~min}$. ATI $(15 \mu \mathrm{L}$, $1 \mathrm{mmol} / \mathrm{L}$ ) and an as-prepared $100 \mu \mathrm{L} \mathrm{NC-dots/AuNPs} \mathrm{solution}$ were introduced into the system, followed by the addition of a $40-\mu \mathrm{L} 0.05 \mathrm{~mol} / \mathrm{L}$ HEPES buffer solution ( $\mathrm{pH} 7.4$ ); the mixed solution was diluted to $300-\mu \mathrm{L}$ with ultrapure water. The mixture was kept at $37^{\circ} \mathrm{C}$ for $10 \mathrm{~min}$ and the fluorescence emission spectra were recorded at an excitation wavelength of $360 \mathrm{~nm}$. The slot widths for excitation was fixed at $2.5 \mathrm{~nm}$ and emission was fixed at $5 \mathrm{~nm}$.

\section{Results and Discussion}

\section{Characterizations of $\mathrm{NC}$-dots}

The NC-dots exhibited strong blue photoluminescence under the illumination of UV light at $365 \mathrm{~nm}$. The UV-vis absorption and emission of the as-prepared NC-dots are given in Fig. 1. The spectrum in the UV-vis absorption region exhibits an obvious absorption peak at around $360 \mathrm{~nm}$, attributable to the $n-\pi *$ transition, and another unconspicuous absorption band at $240 \mathrm{~nm}$ attributable to the $\pi-\pi^{*}$ transition..$^{32,38}$ According to Fig. S1, we can know that NC-dots exhibit a fluorescence emission band centred at $455 \mathrm{~nm}$ with the optimal excitation wavelength of $360 \mathrm{~nm}$. The FT-IR of the NC-dots (Fig. S2) demonstrate that the NC-dots were surrounded by $\mathrm{N}-\mathrm{H}$ groups.

\section{Principle of pesticide detection base on NC-dots/AuNPs sensing} system

The working principle of this biosensor is illustrated in Scheme 1. The NC-dots are mixed with AuNPs firstly to form the a NC-dots "shell" assembly on the surface of the AuNPs through the Au-N interaction. The fluorescence of the NC-dots/ AuNPs assembly is quenched as shown in Fig. 2 (curves a and d). In the absence of paraoxon, the active AChE will catalyze the hydrolysis of ATI into thiocholine, which is positively charged and bears an additional sulfydryl (-SH). Thiocholine can replace the NC-dots on the surface of AuNPs and trigger the 


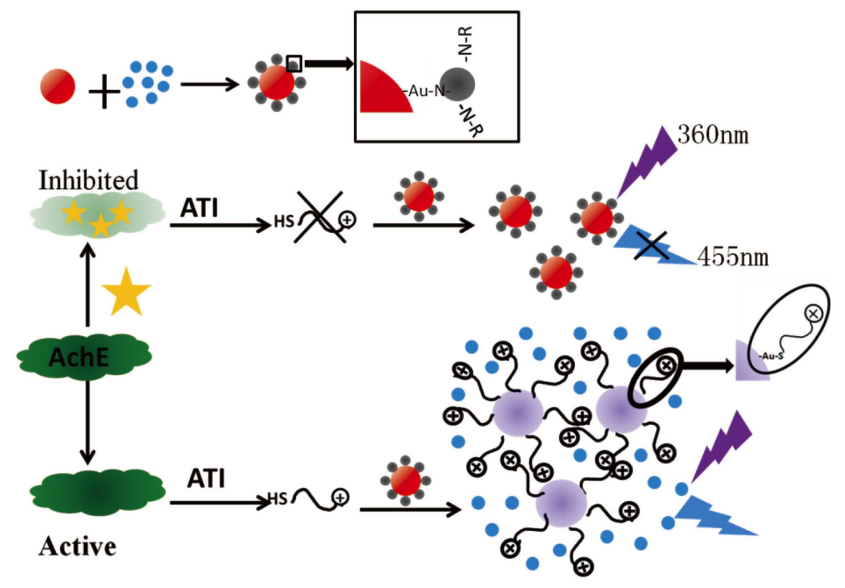

$\star$ : paraoxon :AuNPs $\quad \bullet$ :NC-dots with fluorescence

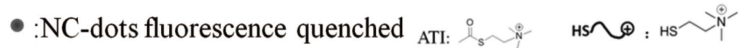

Scheme 1 Schematic illustration of the NC-dots/AuNPs fluorescence assay for the detection of organophosphorus pesticides.

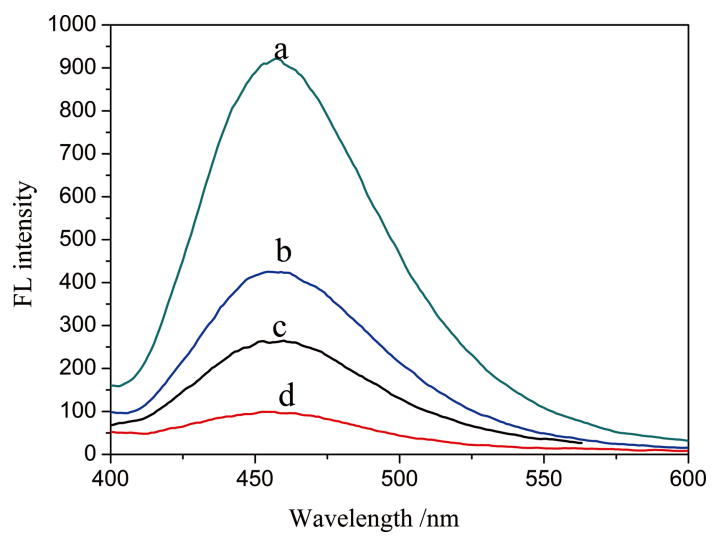

Fig. 2 Fluorescence emission spectra: (a) NC-dots; (b) mixture of NC-dots/AuNPs, ATI, and AChE; (c) mixture of NC-dots/AuNPs, ATI, $\mathrm{AChE}$, and paraoxon; (d) NC-dots/AuNPs. NC-dots, $0.4575 \mathrm{mg} / \mathrm{L}$; AuNPs, $7.5 \mathrm{nmol} / \mathrm{L}$; ATI, $50 \mu \mathrm{mol} / \mathrm{L}$; AChE, $10 \mathrm{mU} / \mathrm{mL}$; paraoxon, $3.0 \times 10^{-6} \mathrm{~g} / \mathrm{L}$.

aggregation of AuNPs, with the fluorescence emission of NCdots recovered (curve b) and a color change of the solution from red wine to black. The desorption of NC-dots is caused by competition between - $\mathrm{SH}$ and nitrogen-containing groups; thiocholine can bind more strongly onto surface of AuNPs than NC-dots. In the presence of paraoxon, the activity of AChE will be inhibited, and the amount of thiocholine generated will be insufficient, so the fluorescence recovery degree of the system is decreased (curve c). The fluorescence of the NC-dots will gradually be quenched with increasing the concentration of paraoxon, and thus making the fluorescence analysis of pesticides feasible. The UV-vis absorption spectra of AuNPs in different states are shown in Fig. S3. The assembly process of NC-dots on the AuNPs does not influence the dispersion of the particles; the absorption peaks at about $519 \mathrm{~nm}$ in the absence (a) and presence (b) of NC-dots are almost identical. Also, the background absorbance between 600 and 700 increase only slightly after the assembly of NC-dots on AuNPs, and the color of the dispersed particle solution is like red wine. In the

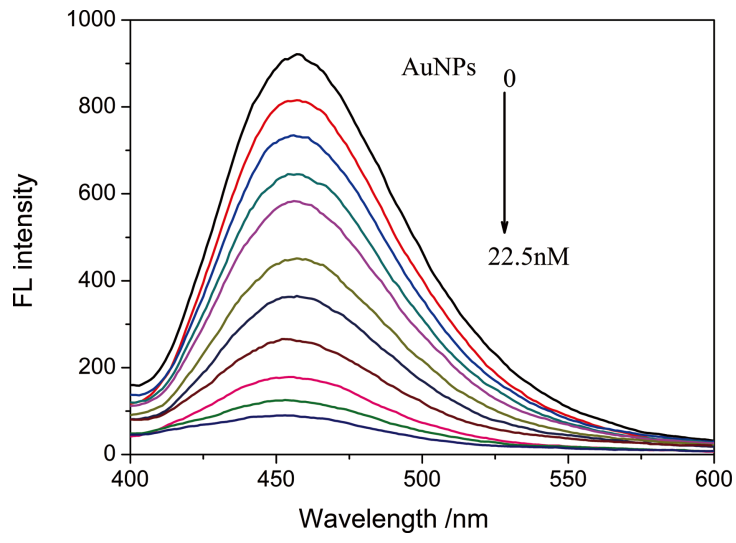

Fig. 3 Emission spectra of NC-dots $\left(C_{\text {NC-dots }}=0.4575 \mathrm{mg} / \mathrm{L}\right)$ in the presence of different amounts of AuNPs ( $C_{\text {AuNPs }}: 0$ to $\left.22.5 \mathrm{nmol} / \mathrm{L}\right)$ in HEPES buffer (10 mmol/L, pH 7.4).

presence of AChE and ATI (curve d), NC-dots/AuNPs are aggregated, and the color of the solution turns to black. According to the UV spectra, the absorption peak at $519 \mathrm{~nm}$ disappeares, and a new peak at about $700 \mathrm{~nm}$ is observed. In the presence of AChE, ATI and paraoxon (curve c), the activity of AChE are inhibited and the peak at $519 \mathrm{~nm}$ reappears with decreasing the peak intensity at about $700 \mathrm{~nm}$, and solution color turns to purple.

The fluorescence of NC-dots is quenched by AuNPs. Figure 3 shows fluorescence spectra of NC-dots $(0.4575 \mathrm{mg} / \mathrm{L})$ in $\mathrm{pH} 7.4$ HEPES buffer in the presence of different amounts of AuNPs. Upon the addition of AuNPs, the fluorescence intensity of NC-dots decreases gradually due to the quenching process. More than $80 \%$ of the fluorescence at $455 \mathrm{~nm}$ is quenched when AuNPs $(7.5 \mathrm{nmol} / \mathrm{L})$ are added to the NC-dots solution. As shown in Fig. S4, the emission spectrum of NC-dots overlaps with the absorbance spectrum of AuNPs to some extent, indicating a potential to produce efficient energy transfer, and the fluorescence quenching of NC-dots shows a FRET mechanism. The fluorescent of NC-dots is quenched by AuNPs.

To further confirm the interaction between NC-dots and AuNPs, TEM assays were performed. The results are displayed in Fig. 4. As shown in Fig. 4A, AuNPs are spherical in shape with a smooth surface and in good dispersion. After mixing with NC-dots, AuNPs are still a dispersion system, but highresolution transmission electron microscopy (HRTEM) displays an impact "shell" on the surface of AuNPs (Fig. 4B). Upon the addition of AChE and ATI, monodispersed NC-dots/AuNPs show a tendency of aggregation, and a large amount of AuNPs clusters appear, while NC-dots are desorbed from the AuNPs, and the "shell" structure on AuNPs disappears (Fig. 4C). However, the aggregation of AuNPs and the desorption of NC-dots are prevented when paraoxon is added to inhibit the activity of AChE (Fig. 4D). As shown in Fig. 4D, an impact NC-dots "shell" on the AuNPs surface appears again. These results are in agreement with the optical spectra and are accordant with the detection mechanism.

Analytical performances and possible mechanism of the biosensing system

Under the optimized conditions, the linear response range of the sensing system was measured. The fluorescence spectra of the NC-dots/AuNPs in the presence of AChE-ATI with different amounts of paraoxon under the optimum conditions are shown 

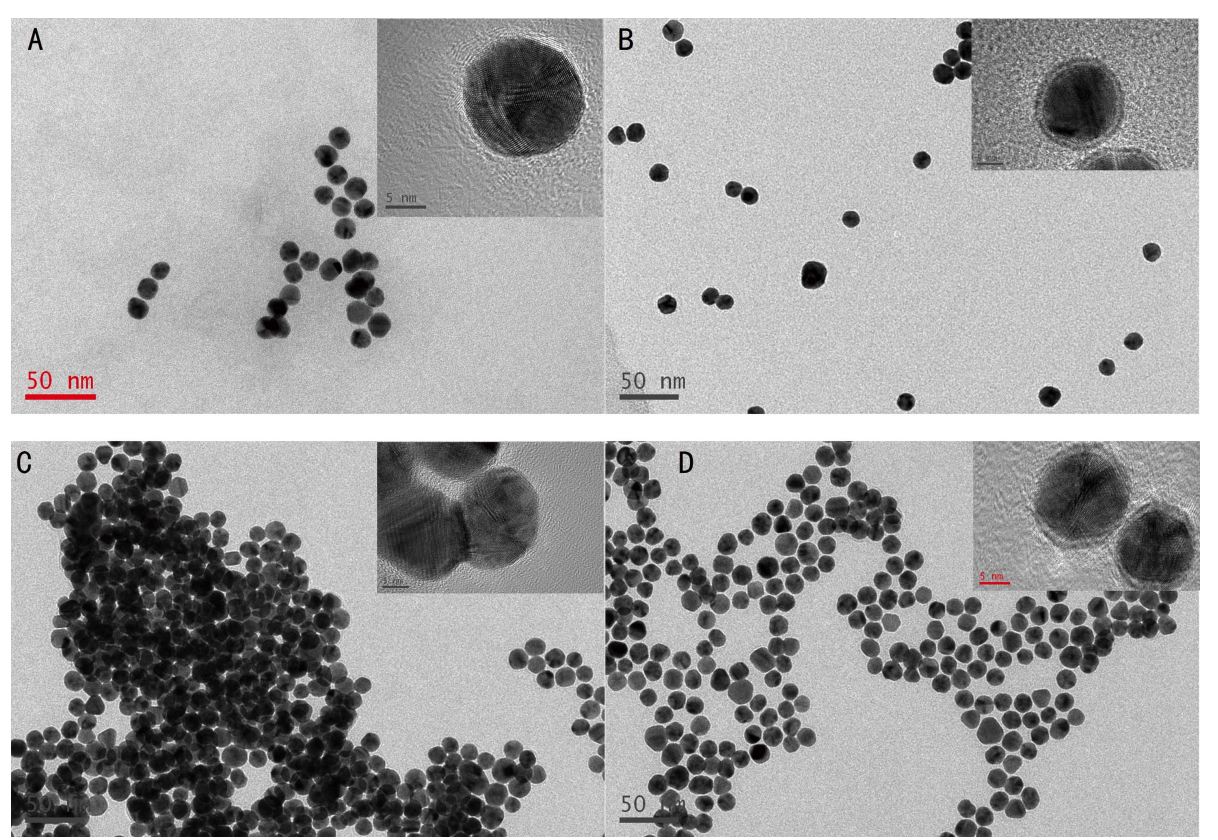

Fig. 4 (A) TEM of AuNPs, (B) NC-dots/AuNPs, (C) NC-dots/AuNPs after adding AChE and ATI, (D) NC-dots/AuNPs by pretreating AChE with the paraoxon. Insets in the panel show the HRTEM of individual AuNPs in each part.
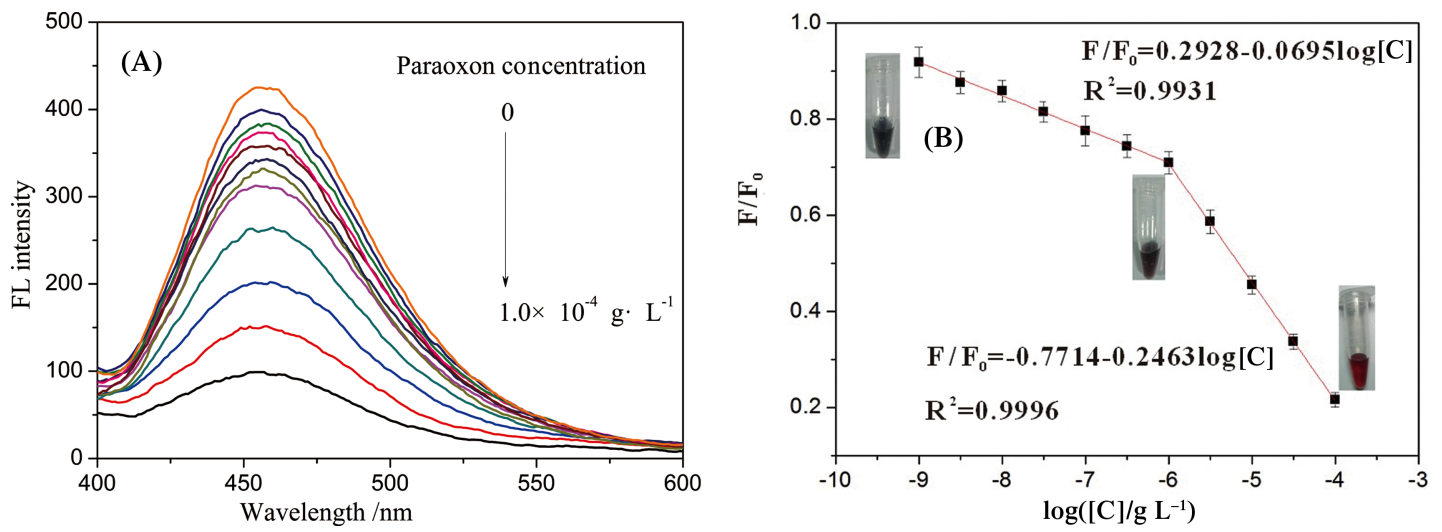

Fig. 5 (A) Fluorescence spectra of NC-dots/AuNPs-ATI-AChE system in the presence of various concentrations of paraoxon. The concentrations of paraoxon (from top to bottom) are $0,1.0 \times 10^{-9}$, $3.0 \times 10^{-9}, 1.0 \times 10^{-8}, 3.0 \times 10^{-8}, 1.0 \times 10^{-7}, 3.0 \times 10^{-7}, 1.0 \times 10^{-6}, 3.0 \times 10^{-6}, 1.0 \times 10^{-5}, 3.0 \times 10^{-5}$, and $1.0 \times 10^{-4} \mathrm{~g} / \mathrm{L}$, respectively. (B) Linear calibration of $F / F_{0}$ versus the logarithm of paraoxon concentrations. The concentrations for AChE, ATI, AuNPs, and NC-dots are the same as those in Fig. 2.

in Fig. 5A. As displayed in this figure, with increasing the concentration of paraoxon, the fluorescence intensity of NC-dots decreased (from top to bottom) gradually. As shown in Fig. 5B, the relative fluorescence intensity of $F / F_{0}\left(F_{0}\right.$ and $F$ are the fluorescence intensity of NC-dots at $455 \mathrm{~nm}$ in the absence and presence of paraoxon, respectively) against the concentrations of paraoxon are plotted; higher concentrations of paraoxon cause less fluorescence recovery.

It is clear that the data points do not follow the same linear trend in the entire concentration range. By a careful examination, we found that there are two linear regions within the concentrations of paraoxon between $10^{-9}$ and $10^{-4} \mathrm{~g} / \mathrm{L}$, as shown in Fig. 5B. The $F / F_{0}-\log [\mathrm{C}]$ relationship is shown to be linear from $1.0 \times 10^{-4}$ to $1.0 \times 10^{-6} \mathrm{~g} / \mathrm{L}\left(3.6 \times 10^{-7}\right.$ to $\left.3.6 \times 10^{-9} \mathrm{~mol} / \mathrm{L}\right)$ with a relatively larger slope than that of the $1.0 \times 10^{-9}$ to
$1.0 \times 10^{-6} \mathrm{~g} / \mathrm{L}\left(3.6 \times 10^{-12}\right.$ to $\left.3.6 \times 10^{-9} \mathrm{~mol} / \mathrm{L}\right)$ region. The linear-regression equation for the low concentration range can be expressed as $F / F_{0}=0.2928-0.0695 \log [\mathrm{C}]$ with a correlation coefficient $\left(R^{2}\right)$ of 0.9931 ; also the equation for the high concentration range was $F / F_{0}=-0.7714-0.2463 \log [\mathrm{C}]$, with a $R^{2}$ of 0.9996 . It is worth noting that the slope obtained in the high-concentration range is three-times more than that obtained in the low-concentration range, and there is an turning point at around $10^{-6} \mathrm{~g} / \mathrm{L}$. These phenomena suggest that there are two different responsive mechanisms in the high and lowconcentration ranges.

According to the principles of the biosensor, in the absence of paraoxon, the active AChE will generate a large amount of thiocholine to substitute the NC-dots on the AuNPs surface. In such a case, the fluorescence intensity will be the strongest. In 


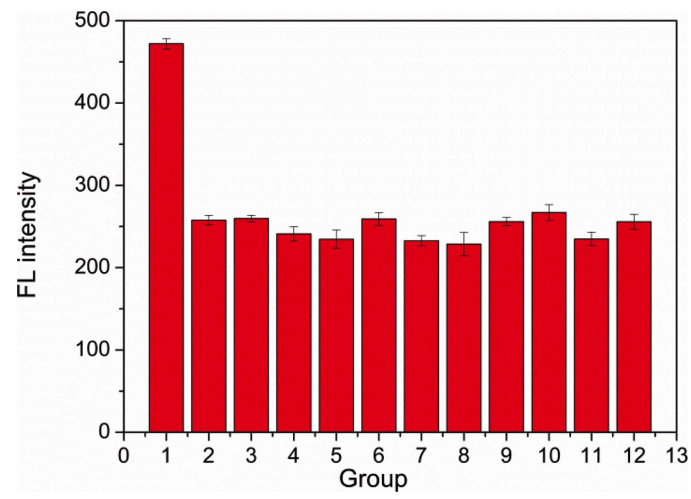

Fig. 6 Fluorescence intensity of NC-dots/AuNPs-ATI-AChE in the presence of paraoxon $\left(3.0 \times 10^{-6} \mathrm{~g} / \mathrm{L}\right)$ premixed with different disruptors $\left(1.0 \times 10^{-4} \mathrm{~g} / \mathrm{L}\right)$. 1, NC-dots/AuNPs-ATI-AChE; 2, control (NC-dots/AuNPs-ATI-AChE-paraoxon); 3 - 12, NC-dots/AuNPsATI-AChE-paraoxon in the presence of disruptors $(3, \mathrm{NaCl} ; 4, \mathrm{KCl}$; $5, \mathrm{MgCl}_{2} ; 6, \mathrm{AlCl}_{3} ; 7, \mathrm{CaCl}_{2} ; 8, \mathrm{ZnCl}_{2} ; 9$, glucose; 10, glycine; 11, L-glutathione; 12, vitamin $\mathrm{C}$ ).

the presence of paraoxon, the activity of $\mathrm{AChE}$ will be inhibited, and the amount of thiocholine is insufficient, so the fluorescence of the NC-dots could not be fully restored. Ideally, in this assay, the fluorescence will be gradually quenched with increasing the concentration of paraoxon. Nevertheless, this ideal state can only occur when the fluorescence change is entirely determined by the extent of thiocholine/NC-dots replacement on the AuNPs surface. In the actual reaction, the aggregation state of AuNPs was another important factor that would influence the fluorescence quenching, especially in the lower concentration range $\left(1.0 \times 10^{-9}\right.$ to $1.0 \times 10^{-6} \mathrm{~g} / \mathrm{L}$ region $)$ Incomplete inhibition would generate a large amount of thiocholine, which could not only replace the NC-dots on the AuNPs surface, but also trigger the aggregation of AuNPs. Within the lower concentration range, the color of the whole system was black or black-gray, and a red shift of absorption peak position could be observed compared with the un-aggregated AuNPs (Fig. S4). Thus, the overlapping between the emission peak of the NC-dots and the absorption peak of the AuNPs was significantly decreased, leading to a weakened FRET effect, and the fluorescence could be partially restored. Due to this fluorescence-recovery the interference of particle aggregation in the $10^{-9}$ to $10^{-6} \mathrm{~g} / \mathrm{L}$ region, the overall fluorescent quenching efficiency of the biosensor dropped a lot. However, during the higher concentration range $\left(10^{-6}\right.$ to $\left.10^{-4} \mathrm{~g} / \mathrm{L}\right)$, the effective inhibition of the AChE generated only a small amount of thiocholine, and the fluorescent change was determined mainly by the extent of thiocholine/NC-dots replacement. Therefore, the response of the biosensor in the high-concentration range was much more sensitive than that of the low-concentration range. The concentration of paraoxon at about $10^{-6} \mathrm{~g} / \mathrm{L}$ was proved to be the turning point to evaluate the existence of fluorescent-recovery interference produced by particle aggregation.

In this assay, the paraoxon was firstly mixed with $\mathrm{AChE}$ for $20 \mathrm{~min}$; then substrate, ATI and NC-dots/AuNPs were added, and left to react for $10 \mathrm{~min}$ before fluorescence detection. Since the reaction time was strictly controlled, another factor that could not be neglected was the catalytic reaction rate of the of the AChE. When the paraoxon concentration was lower than $10^{-6} \mathrm{~g} / \mathrm{L}$, the inhibition was not sufficient, so, within $10 \mathrm{~min}$ most of the ATI molecules were catalyzed into thiocholine due
Table 1 Application of the proposed method for analysis of the apple juice spiked with different amounts of paraoxon

\begin{tabular}{cccc}
\hline Spiked/g L & Experimental value ${ }^{\mathrm{a}} / \mathrm{g} \mathrm{L}^{-1}$ & Recovery, \% & $\mathrm{RSD}, \%$ \\
\hline $5.0 \times 10^{-8}$ & $(4.31 \pm 0.79) \times 10^{-8}$ & 86.1 & 18.2 \\
$5.0 \times 10^{-7}$ & $(4.76 \pm 0.81) \times 10^{-7}$ & 95.3 & 17.1 \\
$5.0 \times 10^{-6}$ & $(4.68 \pm 0.70) \times 10^{-6}$ & 93.5 & 14.9 \\
$5.0 \times 10^{-5}$ & $(5.10 \pm 0.71) \times 10^{-5}$ & 102.0 & 14.0 \\
\hline
\end{tabular}

a. Average of three determinations \pm standard deviation.

to the good enzyme activity. However, the $\Delta F / F_{0}$ versus concentration change of paraoxon was not so significant. However, within the $10^{-4}$ to $10^{-6} \mathrm{~g} / \mathrm{L}$ region, the activity of AChE was well suppressed, and a substantial excess of ATI over AchE emerged. In this case, the $\Delta F / F_{0}$ could obviously reflect the inhibition extent of AchE or concentration change of paraoxon. By combining these three factors, one can understand why there are two linear response regions for this biosensor, and why the slope obtained between $10^{-4}$ to $10^{-6} \mathrm{~g} / \mathrm{L}$ is three-times more than the slope obtained in the $10^{-9}$ to $10^{-6} \mathrm{~g} / \mathrm{L}$ region.

\section{Anti-interference capability of the the system for paraoxon}

In order to evaluate the specificity of the biosensor for the detection of paraoxon, some potentially common existing substances in food samples, such as $\mathrm{NaCl}, \mathrm{KCl}, \mathrm{MgCl}_{2}, \mathrm{AlCl}_{3}$, $\mathrm{CaCl}_{2}, \mathrm{ZnCl}_{2}$, glucose, glycine, L-glutathione, and vitamin $\mathrm{C}$, were examined. Figure 6 shows the fluorescent responses of the biosensor in the absence of paraoxon (1) and in the presence of paraoxon for certain concentration that were mixed with different interferences $(2-12)$. It is very clear that no obvious differences were observed when interfering substance were added. These metal ions and organic matter do not influence the $\mathrm{Au}-\mathrm{N}$ and $\mathrm{Au}-\mathrm{S}$ interaction, and have a negligible interfering effect on the paraoxon determination. Consequently, the results have clearly suggested that the NC-dots/AuNPs based fluorescence biosensor provided excellent specificity and antiinterference capability in paraoxon detection.

\section{Analysis of paraoxon in spiked samples}

To evaluate the practicality of the present method, the fluorescence assay has been applied to detect paraoxon level in apple juice. Certain amounts of paraoxon standard solution were directly spiked into the pretreated apple juice. The analytical results are given in Table 1 . The good recoveries ranging from 86.1 to $102 \%$ with an RSD of ranging from 14.0 to $18.2 \%$ definitely demonstrate the accuracy and reliability of the present fluorescence method for detecting paraoxon in practical applications.

\section{Conclusions}

In summary, a sensitive and selective FRET biosensor for paraoxon detection was developed based on the NC-dots/AuNPs nanoassembly. Compared with previously reported methods, this biosensor is simple and economic, and dose not need any complex synthesis and/or modification to form a stable NC-dots "shell" on a gold surface. Effective fluorescent quenching of NC-dots was obtained through the Au-N interaction. The fluorescent sensor showed excellent sensitivity and good selectivity over that of other disruptors for paraoxon detection with detection limits of $1.0 \times 10^{-9} \mathrm{~g} / \mathrm{L} ; 3.6 \times 10^{-12} \mathrm{~mol} / \mathrm{L}$ ). There were two linear response regions, $1.0 \times 10^{-4}$ to 
$1.0 \times 10^{-6} \mathrm{~g} / \mathrm{L}$ and $1.0 \times 10^{-9}$ to $1.0 \times 10^{-6} \mathrm{~g} / \mathrm{L}$; the slope of the former is three-times more than that of the latter. Three factors, the extent of thiocholine/NC-dots replacement on AuNPs surface, the fluorescent-recovery interference produced by particle aggregation, and the catalytic reaction rate of the AChE, were discussed in detail in Supporting Information, so as to understand the different responsive mechanisms in the high and low-concentration ranges. This study provides a sensitive and rapid detection method for pesticide residues in agricultural products and clinical diagnosis assays.

\section{Acknowledgements}

This work was financially supported by the National Natural Science Foundation of China (No. 21275044, J1210040).

\section{Supporting Information}

This material is available free of charge on the Web at http:// www.jsac.or.jp/analsci/.

\section{References}

1. D. E. Ray, Toxicol. Lett., 1998, 102, 527.

2. G. Aragay, F. Pino, and A. Merkoci, Chem. Rev., 2012, 112, 5317.

3. C. N. Pope, J. Toxicol. Env. Heal. B, 1999, 2, 161.

4. S. Roast, R. Thompson, P. Donkin, J. Widdows, and M. Jones, Water Res., 1999, 33, 319.

5. H. Wang, J. Wang, C. Timchalk, and Y. Lin, Anal. Chem., 2008, $80,8477$.

6. D. Du, S. Chen, J. Cai, Y. Tao, H. Tu, and A. Zhang, Electrochim. Acta, 2008, 53, 6589.

7. H. Chen, X. Zuo, S. Su, Z. Tang, A. Wu, S. Song, D. Zhang, and C. Fan, Analyst, 2008, 133, 1182.

8. C. Xie, H. Li, S. Li, J. Wu, and Z. Zhang, Anal. Chem., 2009, 82, 241.

9. N. Jha and S. Ramaprabhu, Nanoscale, 2010, 2, 806.

10. Y.-H. Won, H. S. Jang, S. M. Kim, E. Stach, M. Ganesana, S. Andreescu, and L. A. Stanciu, Langmuir, 2009, 26, 4320.

11. D. Lu, G. Shao, D. Du, J. Wang, L. Wang, W. Wang, and Y. Lin, Lab Chip, 2011, 11, 381.

12. G. Liu, X. Yang, T. Li, H. Yu, X. Du, Y. She, J. Wang, S. Wang, F. Jin, M. Jin, H. Shao, L. Zheng, Y. Zhang, and P. Zhou, Microchim. Acta, 2015, 182, 1983.

13. D. Liu, W. Chen, J. Wei, X. Li, Z. Wang, and X. Jiang, Anal. Chem., 2012, 84, 4185.
14. J. Guo, H. Li, M. Xue, M. Zhang, X. Cao, Y. Luo, F. Shen, and C. Sun, Food Anal. Method, 2013, 7, 1247.

15. Q. Long, H. Li, Y. Zhang, and S. Yao, Biosens. Bioelectron., 2015, 68, 168.

16. Y. Yi, G. Zhu, C. Liu, Y. Huang, Y. Zhang, H. Li, J. Zhao, and S. Yao, Anal. Chem., 2013, 85, 11464.

17. G. Guan, S. Y. Zhang, Y. Cai, S. Liu, M. S. Bharathi, M. Low, Y. Yu, J. Xie, Y. Zheng, Y. W. Zhang, and M. Y. Han, Chem. Commun., 2014, 50, 5703.

18. T. Yu, T.-Y. Ying, Y.-Y. Song, Y.-J. Li, F.-H. Wu, X.-Q. Dong, and J.-S. Shen, RSC Adv., 2014, 4, 8321.

19. K. Zhang, T. Yu, F. Liu, M. Sun, H. Yu, B. Liu, Z. Zhang, H. Jiang, and S. Wang, Anal. Chem., 2014, 86, 11727.

20. Y. He, B. Xu, W. Li, and H. Yu, J. Agric. Food Chem., 2015, 63, 2930.

21. X. Yan, H. Li, X. Wang, and X. Su, Talanta, 2015, 131, 88.

22. X. Wang, T. Hou, S. Dong, X. Liu, and F. Li, Biosens. Bioelectron., 2016, 77, 644.

23. K. Qian, Q. Deng, G. Fang, J. Wang, M. Pan, S. Wang, and Y. Pu, Biosens. Bioelectron., 2016, 79, 359.

24. G. Guan, L. Yang, Q. Mei, K. Zhang, Z. Zhang, and M. Y. Han, Anal. Chem., 2012, 84, 949.

25. X. Meng, J. Wei, X. Ren, J. Ren, and F. Tang, Biosens. Bioelectron., 2013, 47, 402.

26. P. Kumar, K. H. Kim, and A. Deep, Biosens. Bioelectron., 2015, 70, 469.

27. G. Aragay, F. Pino, and A. Merkoci, Chem. Rev., 2012, 112, 5317.

28. P. A. Souza Tette, L. Rocha Guidi, M. B. de Abreu Gloria, and C. Fernandes, Talanta, 2016, 149, 124.

29. Y. Wang, L. Xiong, F. Geng, F. Zhang, and M. Xu, Analyst, 2011, 136, 4809.

30. A. Zhao, Z. Chen, C. Zhao, N. Gao, J. Ren, and X. Qu, Carbon, 2015, 85, 309.

31. H. Dai, Y. Shi, Y. Wang, Y. Sun, J. Hu, P. Ni, and Z. Li, Sens. Actuators, B, 2014, 202, 201.

32. F. Du, F. Zeng, Y. Ming, and S. Wu, Microchim. Acta, 2013, 180, 453.

33. Z. Z. Chen, L. Cai, M. Y. Chen, Y. Lin, D. W. Pang, and H. W. Tang, Biosens. Bioelectron., 2015, 66, 95.

34. Z. Qian, L. Chai, C. Tang, Y. Huang, J. Chen, and H. Feng, Anal. Chem., 2015, 87, 2966.

35. Y. Yan, J. Sun, K. Zhang, H. Zhu, H. Yu, M. Sun, D. Huang, and S. Wang, Anal. Chem., 2015, 87, 2087.

36. J. Deng, Q. Lu, Y. Hou, M. Liu, H. Li, Y. Zhang, and S. Yao, Anal. Chem., 2015, 87, 2195.

37. K. C. Grabar, R. G. Freeman, M. B. Hommer, and M. J. Natan, Anal. Chem., 1995, 67, 735.

38. Z. Gao, Z. Lin, X. Chen, H. Zhong, and Z. Huang, Anal. Methods, 2016, 8, 2297. 